\title{
ARTICLE OPEN \\ Predictive value of genomic screening: cross-sectional study of cystic fibrosis in 50,788 electronic health records
}

\author{
J. P. Sugunaraj (D) ${ }^{1}$, H. M. Brosius ${ }^{1}$, M. F. Murray ${ }^{2}$, K. Manickam ${ }^{3}$, J. A. Stamm ${ }^{1}$, D. J. Carey ${ }^{4}$ and U. L. Mirshahi (i) ${ }^{4}$
}

Doubts have been raised about the value of DNA-based screening for low-prevalence monogenic conditions following reports of testing this approach using available electronic health record (EHR) as the sole phenotyping source. We hypothesized that a better model for EHR-focused examination of DNA-based screening is Cystic Fibrosis (CF) since the diagnosis is proactively sought within the healthcare system. We reviewed CFTR variants in 50,778 exomes. In 24 cases with bi-allelic pathogenic CFTR variants, there were 21 true-positives. We considered three cases "potential" false-positives due to limitations in available EHR phenotype data. This genomic screening exhibited a positive predictive value of $87.5 \%$, negative predictive value of $99.9 \%$, sensitivity of $95.5 \%$, and a specificity of $99.9 \%$. Despite EHR-based phenotyping limitations in three cases, the presence or absence of pathogenic CFTR variants has strong predictive value for CF diagnosis when EHR data is used as the sole phenotyping source. Accurate ascertainment of the predictive value of DNA-based screening requires condition-specific phenotyping beyond available EHR data.

npj Genomic Medicine (2019)4:21 ; https://doi.org/10.1038/s41525-019-0095-6

\section{INTRODUCTION}

Attempts to model genomic screening for long QT syndrome (LQTS) and arrhythmogenic right ventricular cardiomyopathy (ARVC), using electronic health record (EHR) as the lone source of phenotypic data has raised questions about the value of genomic screening more broadly in the accurate identification of genetic disease risk in low-prevalence cohorts. ${ }^{1-4}$

Cystic fibrosis (CF), like LQTS and ARVC, is relatively uncommon but it does not meet the NIH definition of a rare disease. ${ }^{5}$ The reported prevalence of these three conditions range from $\sim 1$ in 1250 to 1 in $3200 .^{6-8}$

However, CF is distinct from LQTS and ARVC in that there has been over 20 years of extensive proactive CF diagnostic efforts to identify cases in adult, pediatric, and newborn care. These CF diagnostic efforts, incorporating both genetic and non-genetic diagnostic testing, have led to standardized diagnostic approaches to suspected cases of CF. ${ }^{9}$ As a result, the EHR of patients in major healthcare systems, contain a wealth of data from the diagnostic workups of patients for whom either clinical or laboratory findings have raised suspicion of the diagnosis. ${ }^{10}$

CF is an autosomal recessive disorder that affects one in 3200 in the United States and is caused by bi-allelic pathogenic variants (either homozygous or compound heterozygous pathogenic alleles) in the cystic fibrosis transmembrane conductance regulator (CFTR) gene. ${ }^{11-13}$ The most common pathogenic CFTR allele, F508del, accounts for $~ 70 \%$ of known CF-causing alleles. ${ }^{14}$

As non-DNA-based ascertainment is the first step toward diagnosis in this low-prevalence monogenic condition, CF provides a reasonable use case for examining the predictive value of DNA-based screening when available EHR data is the lone phenotypic source for case confirmation. Universal newborn screening for CF has been in place in the United states since
2010; all states use immunoreactive trypsinogen (IRT) as the initial screen followed by either repeat IRT (IRT/IRT) or DNA-based testing (IRT/DNA) as the follow-up screen. ${ }^{15}$ Separate from IRTbased newborn screening, a distinct second prompt for CF diagnosis is the presence of symptomatic disease., ${ }^{9,16}$ In either scenario, CFTR genetic testing has been employed as part of the diagnostic follow-up to another prompt for more than 20 years.

"Genome-first" describes a screening process that starts with detection of pathogenic DNA variants independent of any demographic or clinical information. ${ }^{17}$ Genome-first approaches to case identification of monogenic disease are increasingly feasible in large cohorts with genomic sequence data. ${ }^{18-20}$ Despite promising data regarding the use of this approach for two conditions with observed screen positive rates of 1 in 190 to 1 in 256 (i.e. BRCA1/2 related cancer risk and Familial Hypercholesterolemia), the efforts cited above using genomic screening in this manner to identify less prevalent monogenic conditions have demonstrated suboptimal performance characteristics for case identification via this approach. ${ }^{1,2}$

A genome-first approach to case identification for autosomal recessive diseases in general, and to CF specifically, has not been previously examined in a large sequenced cohort. Given the existence of available diagnostic approaches and the anticipated robust diagnostic workup data of suspected cases in the EHR, we hypothesized that modeling a genotype-first screening approach to CF case identification using EHRs would perform better than previous efforts in LQTS and ARVC.

In this manuscript, we report the results of applying a genotypefirst approach to CF case identification within the DiscovEHR dataset that links de-identified exome sequence data with participant's de-identified EHR data. ${ }^{21}$ The data for this study came from 50,778 adult and pediatric participants in the

\footnotetext{
${ }^{1}$ Department of Pulmonary and Critical Care Medicine, Geisinger, Danville, PA, USA; ${ }^{2}$ Department of Genetics, Yale School of Medicine, New Haven, CT, USA; ${ }^{2}$ Division Genetic \& Genomic Medicine, Nationwide Children's Hospital, Columbus, OH, USA and ${ }^{4}$ Department of Molecular and Functional Genomics, Geisinger, Danville, PA, USA

Correspondence: U. L. Mirshahi (umirshahi1@geisinger.edu)

These authors contributed equally: J. A. Stamm, D. J. Carey, U. L. Mirshahi
}

Received: 31 May 2019 Accepted: 14 August 2019

Published online: 04 September 2019 


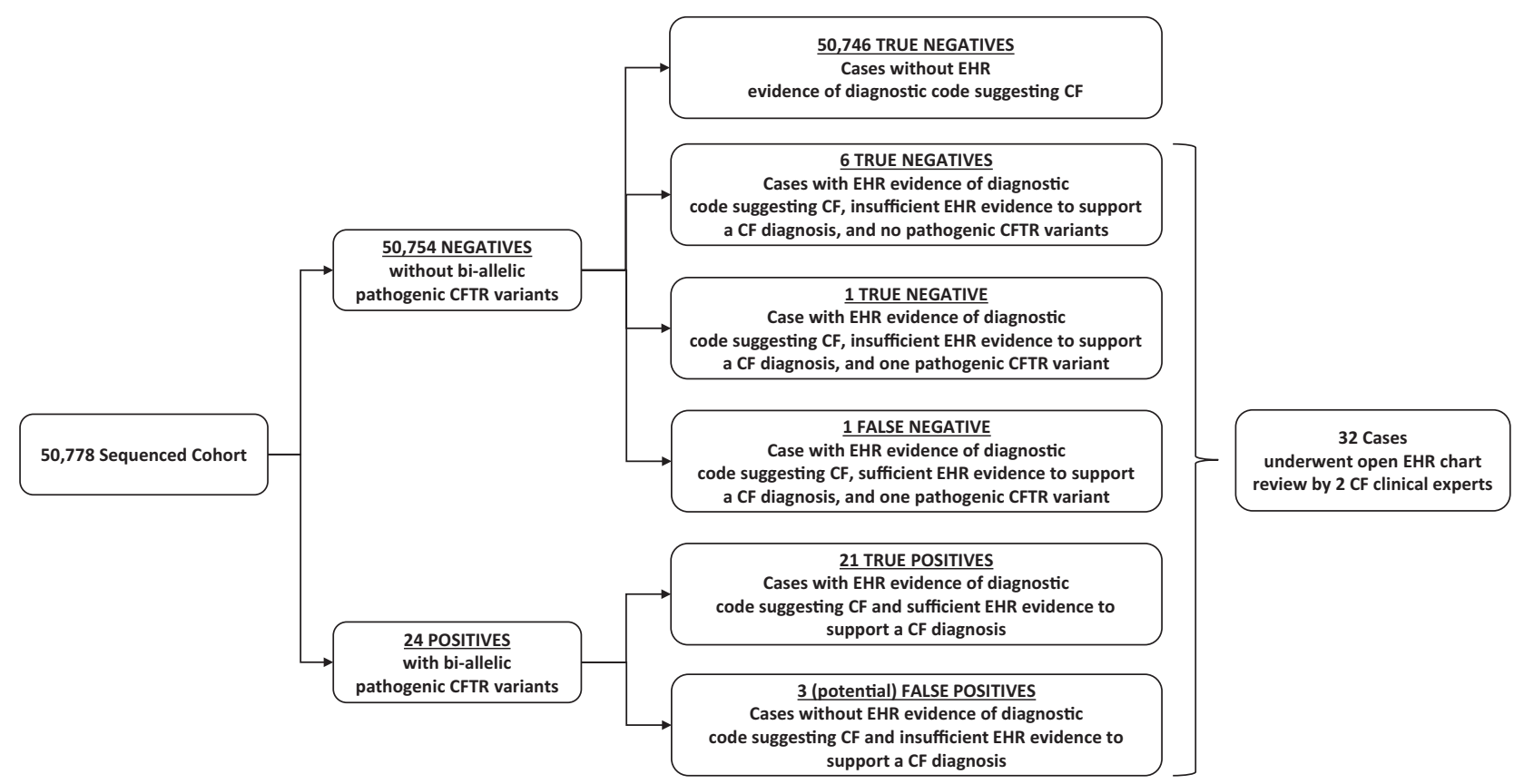

Fig. 1 EHR-based Genomic Screening for CF. In a database of 50,778 participants, there were 50,754 negatives, namely cases without bi-allelic pathogenic CFTR variants, and 24 positives, namely cases with such variants. Amongst the negatives, eight had EHR data suggesting a CF diagnosis, six of those had no CFTR pathogenic variants, and two had a single pathogenic variant. Open chart review concluded that one of the heterozygotes was a false negative and one was a true negative. The remainder of the negatives, those without bi-allelic CFTR variants or EHR data consistent with CF were also considered true negatives. Open chart review was pursued for all 24 positive cases. The diagnosis of CF was confirmed in 21 cases. In the remaining three cases the diagnosis could not be confirmed due to insufficient available evidence

DiscovEHR dataset recruited through the Geisinger MyCode Community Health Initiative (MyCode) in Pennsylvania. IRTbased newborn screening for CF began in Pennsylvania in 1995; therefore, any documented cases in individuals older than 23 years in Pennsylvania would be expected to have almost exclusively occurred through clinical presentations with signs or symptoms.

\section{RESULTS}

The DiscovEHR WES database $(n=50,778)$ was queried for CFcausing variants, defined as those variants identified in the CFTR2 database as CF-causal, and variants identified as pathogenic or likely pathogenic in the ClinVar database.

\section{Genome-first positives}

The exome sequence database query identified 24 cases with biallelic CF-causing CFTR variants (Fig. 1). Expert EHR review confirmed the diagnosis of CF in 21 individuals, who were then labeled true positives (TP); CF could not be confirmed definitively in three individuals (Table 1). This was due to insufficient evidence for, rather than evidence against, a CF diagnosis; these individuals were therefore labeled as "possible" false positives (FP).

\section{Genome-first negatives}

The EHR-associated diagnostic codes for the 50,754 without biallelic pathogenic CFTR variants were queried. Two cases from this group had ICD codes consistent with a CF diagnosis and a monoallelic CFTR pathogenic variant (Fig. 1). These cases underwent expert chart review which confirmed the diagnosis of CF in 1 who was then labeled a false negative (FN). Chart review concluded that the second case has CFTR-Related Metabolic Syndrome (CRMS) variant (Table 1) and was then labeled a true negative (TN). Six individuals had diagnosis codes for CF in EHR but did not harbor any pathogenic CFTR variants. Chart review indicated no evidence of CF and were labeled as true negatives. The remainder of the cases without bi-allelic CFTR variants and no ICD codes consistent with a CF diagnosis were also labeled as TN.

Genetic variants in charts undergoing open review

Table 2 displays the details of the 14 CFTR variants relevant to these 26 cases. The F508del variant accounted for $73 \%$ of the 52 CFTR alleles. The cases discussed above that were ultimately identified as TN and FN were CFTR compound heterozygotes with F508del and S1235R (TN, with CRMS) and F508del and R1239S in the (FN). Importantly the S1235R variant has been judged nonpathogenic in both CFTR2 and ClinVar, and R1239S has not been evaluated for inclusion in either database. In addition to evidence of R1239S pathogenicity in case \#22, there is additional bioinformatic support for the pathogenicity of this variant from our studies (see Table S2) and others. ${ }^{22}$

The three possible FP cases are all F508del compound heterozygotes. The variant L206W found with F508del in two unrelated men over 60 years of age is considered pathogenic in both CFTR2 and ClinVar databases. The third possible FP case was a F508del/Q1476X compound heterozygote in a woman over 70 years of age; the Q1476X allele is described in ClinVar as pathogenic but is not present in CFTR2. Chart review documented that this patient underwent clinical CFTR testing in 2009 that revealed only the F508del heterozygous state. It was further noted that this patient's clinical genetic testing at that time consisted of a limited evaluation for 23 known pathogenic variants; this evaluation was carried out 4 years prior to the documentation of Q1476X as a pathogenic variant in 2013 in ClinVar (see Table 2).

Data concordance in clinical and research CFTR variant results The concordance of clinical CFTR test results from individuals managed in Geisinger CF clinic and compared to exome sequencing results is displayed in Fig. 2. The 20 evaluable cases had $100 \%$ concordance. 


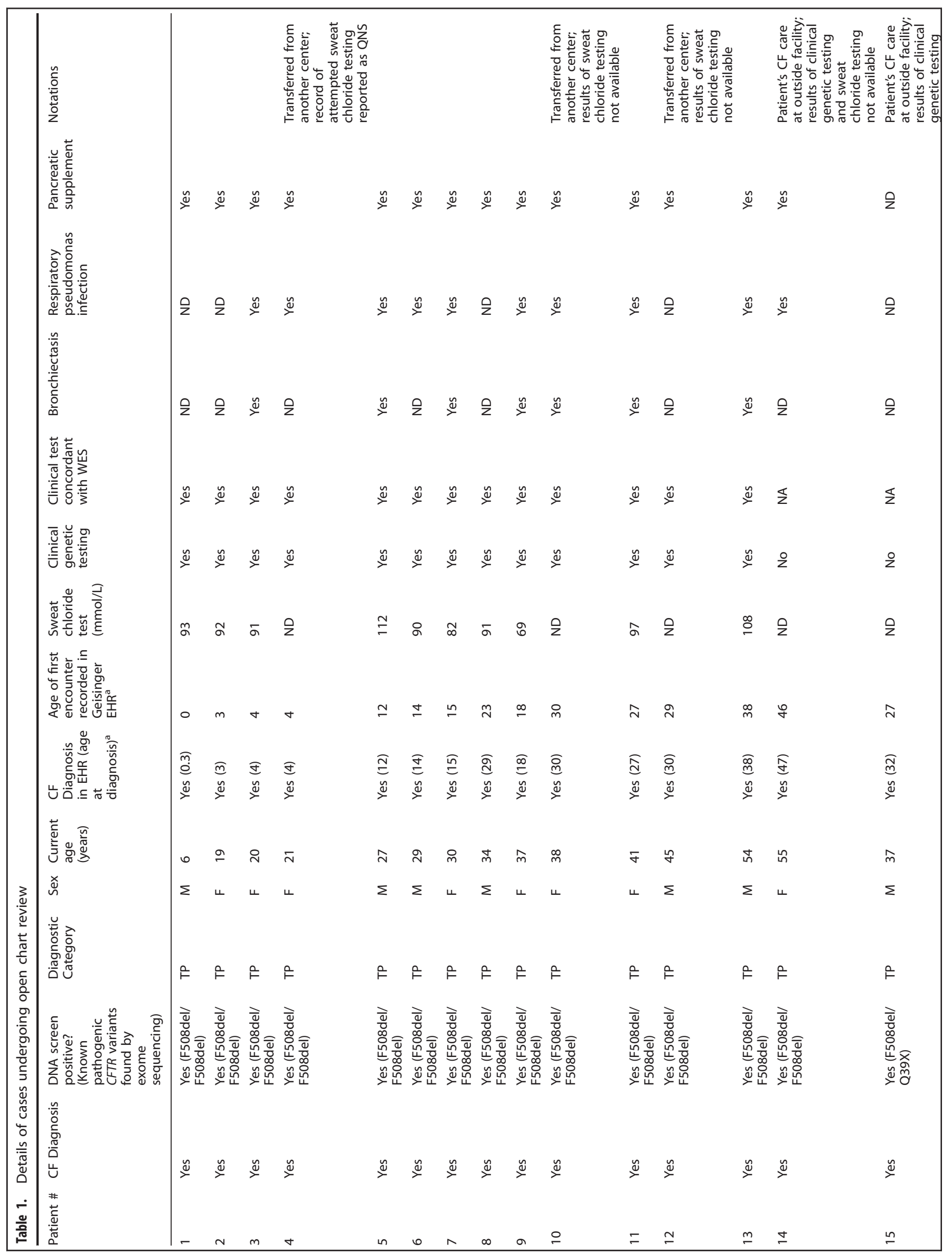




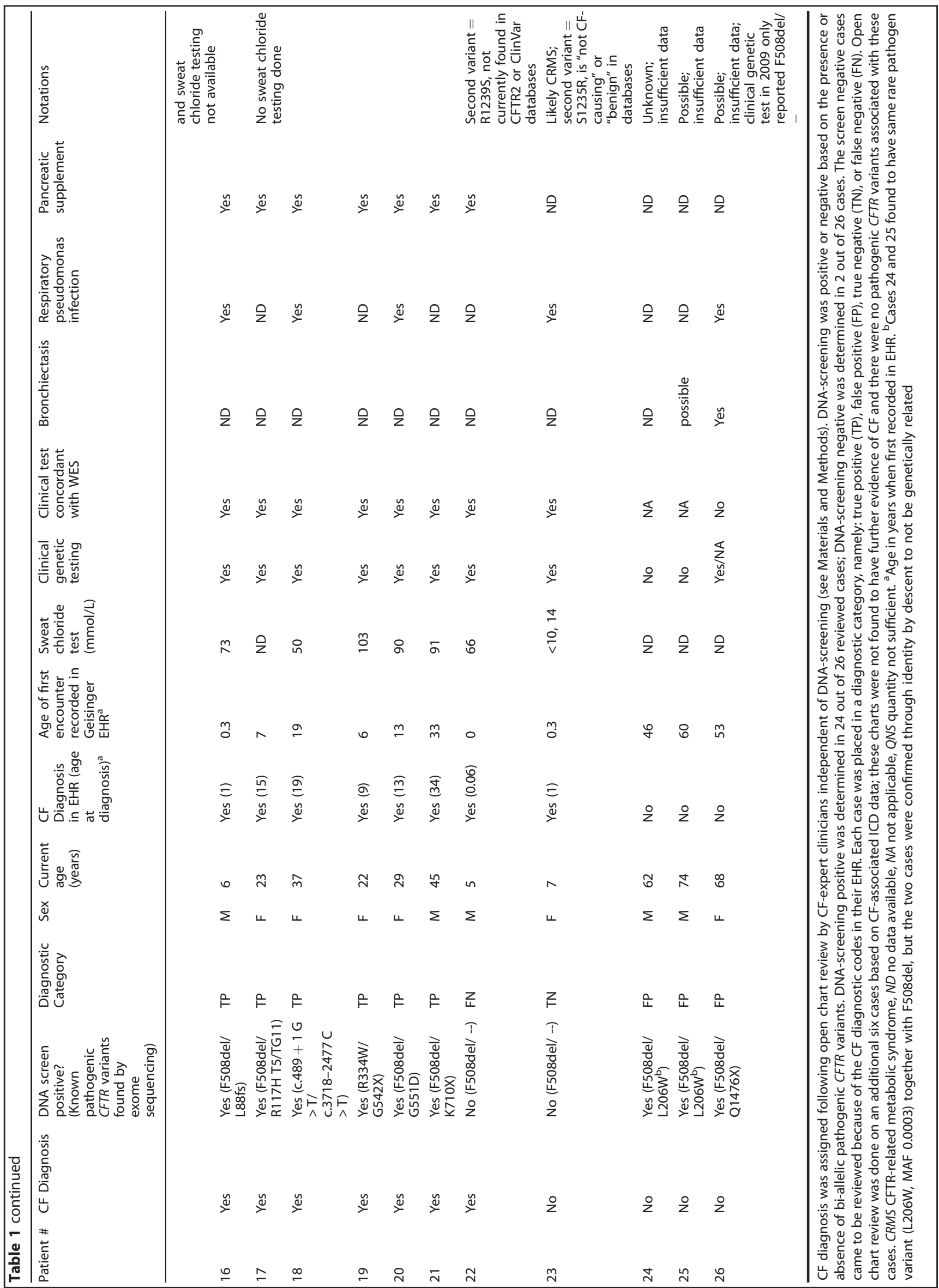




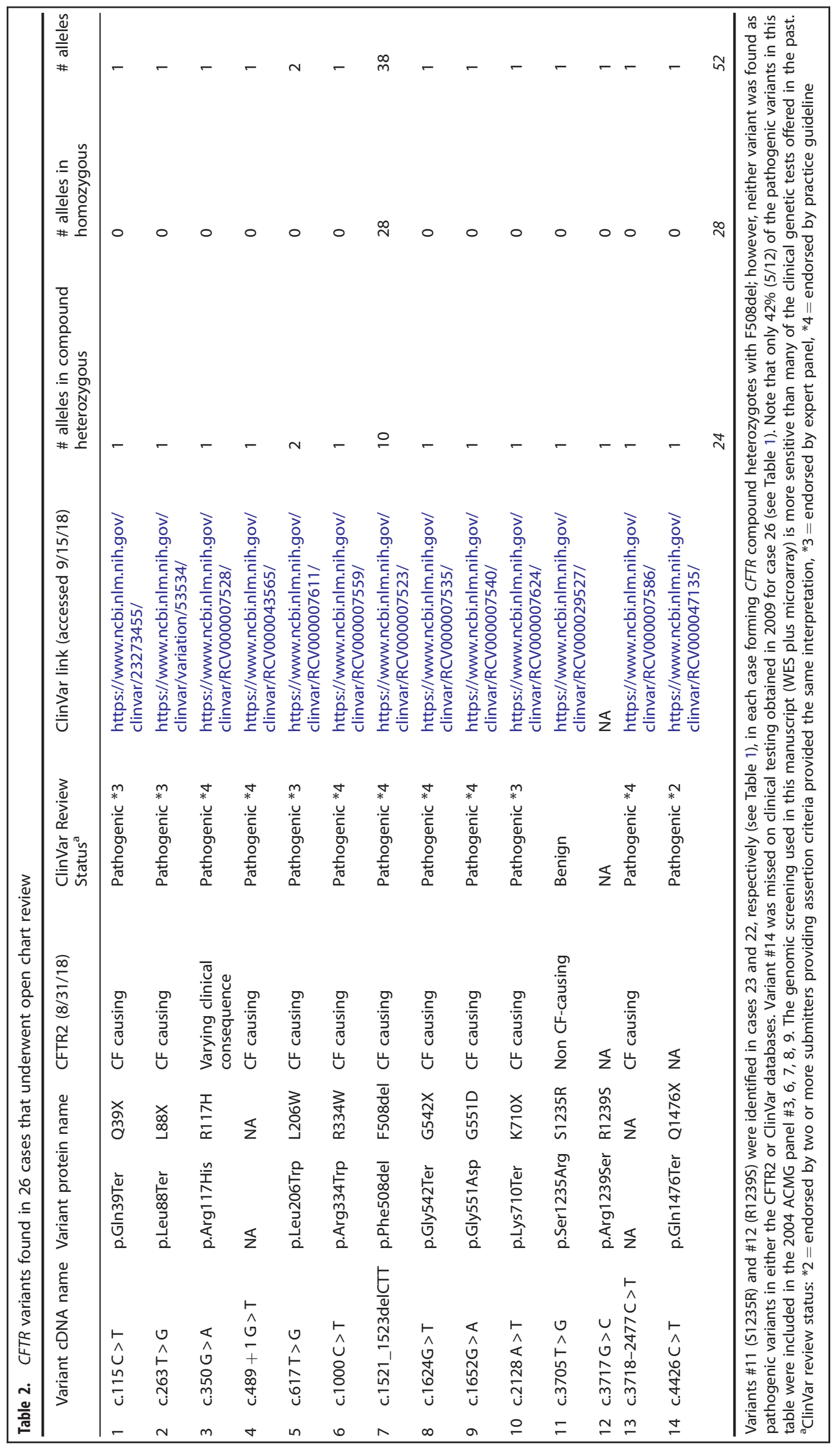




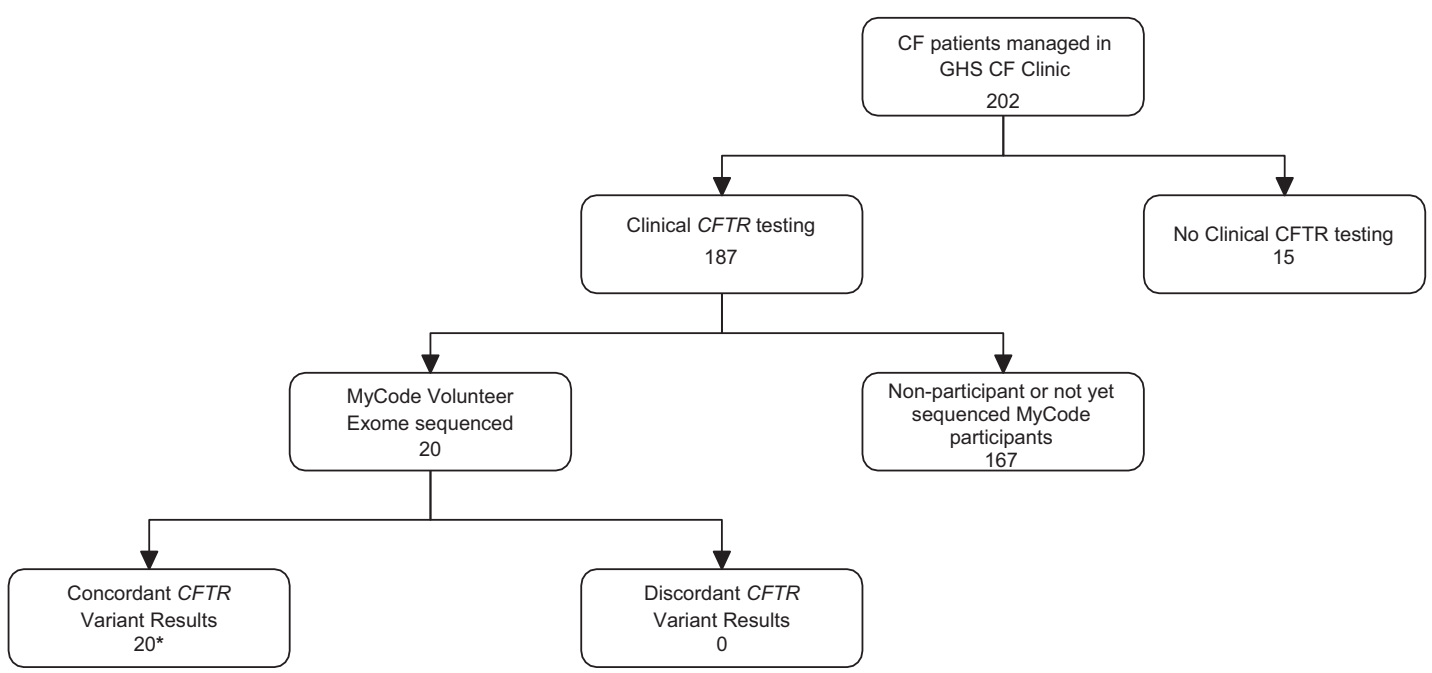

Fig. 2 Data concordance in clinical and research CFTR variant results. Twenty of the 22 confirmed CF patients in the cohort also received their care in the Geisinger CF clinical program and had clinical CF genetic testing results available in their EHR. In the 20 cases with DNA data from both sources there was $100 \%$ pathogenic variant calling concordance

Table 3. Screening predictive statistics

\begin{tabular}{|c|c|c|}
\hline & \multicolumn{2}{|c|}{$\begin{array}{l}\text { Diagnostic } \\
\text { category }\end{array}$} \\
\hline \multicolumn{2}{|l|}{ ES positive, $n(\%)$} & $24(0.05)$ \\
\hline $\mathrm{CF}$ & TP & 21 \\
\hline No CF & $\mathrm{FP}$ & $3^{a}$ \\
\hline \multicolumn{2}{|l|}{ ES negative, $n(\%)$} & $50,754(99.95)$ \\
\hline No CF & TN & 50,753 \\
\hline $\mathrm{CF}$ & $\mathrm{FN}$ & 1 \\
\hline \multicolumn{2}{|l|}{ Sensitivity, \% (95\% Cl) } & $95.5(77.2-99.9)$ \\
\hline \multicolumn{2}{|l|}{ Specificity, \% (95\% Cl) } & 99.99 (99.98-100.00) \\
\hline \multicolumn{2}{|l|}{ PPV, \% (95\% Cl) } & $87.5(69.2-95.6)$ \\
\hline \multicolumn{2}{|l|}{ NPV, \% $(95 \%$ Cl) } & $99.99(99.98-100.00)$ \\
\hline \multicolumn{2}{|l|}{ Accuracy, \% (95\% Cl) } & $99.99(99.98-100.00)$ \\
\hline \multicolumn{2}{|l|}{$\begin{array}{l}\text { Disease prevalence, \% } \\
(95 \% \mathrm{Cl})\end{array}$} & $0.04(0.03-0.07)$ \\
\hline \multicolumn{3}{|c|}{$\begin{array}{l}\text { Individuals with or without CF are categorized as TP, FP, TN, or FN based on } \\
\text { exome sequence variants previously classified as pathogenic. Screening } \\
\text { predictive statistics are calculated as described in Methods. ES exome } \\
\text { sequence, CF cystic fibrosis, TP true positive, FP false positive, TN true } \\
\text { negative, FN false negative, PPV positive predictive value, NPV negative } \\
\text { predictive value, } 95 \% \text { CI } 95 \% \text { confidence interval. "a potential" false positive } \\
\text { - insufficient EHR data to rule in or rule out diagnosis }\end{array}$} \\
\hline
\end{tabular}

Predictive value of exome sequencing

Using the TP, FP, TN, and FN designations described above (displayed in Fig. 1 and Table 3), we calculated positive predictive value of $87.50 \%$ (69.23 to 95.61$)$, negative predictive value of $99.99 \%$ (99.98-100.00), sensitivity of $95.45 \%$ (77.16-99.88), and a specificity of $99.99 \%$ (99.98-100.00).

\section{DISCUSSION}

CF cases in the MyCode Cohort

In the United States, the prevalence of CF in individuals of northern European background is 1:3200 (or 0.03\%), with a carrier frequency of one in 28 individuals. ${ }^{6}$ The 22 confirmed CF cases in this MyCode cohort yields an empiric prevalence of 1:2300 (or $0.04 \%$ ) individuals with $\mathrm{CF}$, and we observed a carrier rate of one in 25 individuals (data not shown).

The positive predictive value (PPV) of screening tests are known to be influenced by the prevalence of the disease in the population that is being screened; more prevalent disease tends to correlate with higher PPV. ${ }^{23}$ It is therefore noteworthy that the population prevalence of CF, LQTS, and ARVC are estimated to be: CF 1:3200, LQTS 1:2500, and AVRC 1:1250. ${ }^{6-8}$ Although the authors did not calculate PPV in their manuscripts, a liberal estimate of PPV using the same definitions for EHR-based TP and FP would be $3.2 \%$ for LQTS and 0\% for ARVC. ${ }^{1,2}$ A PPV of $87.5 \%$ was found for CF in our study.

This study carried out a genome-first DNA-based retrospective analysis of clinical data in a cohort with an average of 14 years of EHR data and an average age of 63 years. In this setting we demonstrated excellent predictive value for genetic screeningbased identification of CF.

Newborn screening for CF is usually based on IRT, a non-DNAbased test. Kloosterboer et al. reported a sensitivity of $80.2 \%$ for the IRT/IRT screening algorithm. ${ }^{24}$ There have been numerous strategies proposed to improve the performance characteristics of the newborn CF screen. ${ }^{25}$ Vernooij-van Langen et al. ${ }^{26}$ carried out screening in 145,499 newborns in the Netherlands. The strategy that performed the best used two non-DNA screens (IRT and pancreatitis-associated-protein) followed by a two DNA screens (panel and sequencing) to get a sensitivity of $95.0 \%$, a specificity of $100 \%$ and a PPV of $87.5 \%$. The current study using DNA sequencing in a non-newborn cohort had a sensitivity, specificity, and PPV similar to the Vernooij-van Langen study.

Of the 26 potential cases reviewed with DNA data, 22 were classified as definite CF, three were not classified as CF due to insufficient data, and one was classified as CRMS. The individual with CRMS, was judge a TN. This case had absence of bi-allelic pathogenic CFTR variants and an EHR showing a negative sweat test result as part of a diagnostic CF workup. So although EHR diagnostic coding included a CF diagnosis, that has been revised to CRMS. It is noteworthy that the DiscovEHR exome sequence data, array genotype data, and clinical genetic tests results, when available, were completely concordant.

The exome sequence data identified three possible previously undiagnosed CF cases that were conservatively judged FP (see evidence support pathogenicity for these variants with milder disease phenotypes in Bioinformatic Narrative in the supplements). 
In one of these individuals there had been clinical suspicion of CF and a record of completed clinical genetic testing for CF at an outside institution. The other two (cases 24 and 25) had no evidence of clinical testing for CF in their EHR, but exome sequencing identified bi-allelic pathogenic variants (F508del and L206W), which have been classified as a CF-causing combination in both the CFTR2 and ClinVar databases.

Improving variant databases

This work relied on the existence of robust variant interpretation databases, and the importance of maintaining and expanding these datasets was highlighted by two of the cases. The clinical testing of case 26 with the heterozygous combination of F508del and Q1476X in 2009 failed to identify a second pathogenic allele; her second pathogenic allele identified in this study was first entered into public data in 2013.

One previously not observed variant that we encountered was R1239S; this variant was found in combination with F508del in case 22 (designated FN). Designation of R1239S as a newly recognized pathogenic variant is supported by our patient's CF diagnosis as well as the bioinformatics analysis in Table S2, and its addition to ClinVar through this work may help future variant interpretation.

$\mathrm{CF}$ is a disease that is most prevalent in people of European ancestry, and the MyCode cohort has 98\% European-Americans (see Table S1). It will be important to seek replication of our findings for CFTR-CF in more diverse cohorts where there is a greater potential for additional pathogenic variants not currently in the databases.

\section{Modeling genome-first population screening using EHRs}

Growing enthusiasm for using DNA-based screening is tempered by legitimate concerns that this could lead to over-diagnosis and over-estimates of risk. ${ }^{27}$ As discussed above, some early attempts to use DNA-based screening of EHR data in cardiac genetic conditions appear to lend support to these concerns. However, the landmark publications on LQTS and AVRC highlight significant limitations linked to those conditions, including: (1) expert disputes over what to interpret as pathogenic genetic variants, (2) a lack of targeted condition-specific diagnostic evaluations in the EHR data associated with cases presenting with partial phenotypes (i.e. minor diagnostic criteria), and (3) the likelihood that we currently have an incomplete understanding of conditionspecific genotype-phenotype correlations (i.e. questions of critical environmental factors for ARVC)., ${ }^{1,2}$

With regard to these limitations in the setting of these two cardiogenetic conditions, the first is being overcome through efforts such as ClinVar and ClinGen, ${ }^{28}$ the second can be overcome in clinical settings where patients are called back for more extensive phenotyping, ${ }^{29}$ and the third will require more research into basic genotype-phenotype correlation. ${ }^{2}$

In the case of CF, examined in this work, the limitations observed in the LQTS and ARVC examples are largely overcome through the use of: expert consensus on pathogenic CFTR variant calls, a clinical data source (i.e. Geisinger's EHR) where comprehensive diagnostic phenotyping and clinical genetic testing of suspected cases is common, and a gene-disease example with clearly established diagnostic criteria that are routinely interrogated by expert clinicians in suspected cases. In this setting there is little evidence of over-estimate of CF risk given the finding that $87.5 \%$ (21 out of 24 ) of those who screened positive had confirmed CF, and there is little expectation of over-diagnosis since the remaining cases who lack sufficient diagnostic evidence could potentially undergo a clinical evaluation and secondary screening with sweat chloride testing to reach a clear conclusion.

Prior to this CF example, given the data on LQTS and AVRC, it would have been tempting to conclude that poor PPVs were inescapable when pursuing population-based DNA-screening for monogenic disease risk in unselected patient groups. However, since this work demonstrates that a desirable PPV can be attained for $C F$, further research focused on a deeper examination of the factors surrounding these three conditions that result in dramatic differences in PPV should be pursued.

CF is a particularly well-studied monogenic condition. CF is a recessively inherited disorder with nearly $100 \%$ penetrance in homozygous or compound heterozygous individuals. Proactive efforts aimed at case identification and diagnosis have occurred within healthcare for more than two decades. A large number of pathogenic variations in the CFTR gene are known, the molecular consequences of those changes are understood, and expertly curated publically available databases containing these variants exist. The sweat chloride test has been in use for 60 years. ${ }^{9,30,31}$ This routinely employed test provides non-DNAbased laboratory evidence of CFTR dysfunction and can be employed to confirm CF diagnoses. CF is clinically distinct without commonly encountered phenocopies. ${ }^{32}$ The range of clinical manifestations of CF including the mild end of the spectrum is well studied.

Together these features have allowed us to test genome-first screening through a cross-sectional study design using the Geisinger EHR, which has been in place for over 20 years, as the sole clinical data source.

With this design and in this setting, we were able to measure both the PPV and the NPV, as well as the sensitivity and specificity of using DNA variant detection as a primary screening technique. Our strict definition of FP led to 3 FP cases amongst 24 positives, even though 2 of those 3 are probable CF and would likely be converted to TP on follow-up evaluation. Therefore, the measured PPV of $87.5 \%$ for genome-first case detection of CF in this cohort is perhaps mildly suppressed by our retrospective EHR-based study design and demonstrates the need for condition-specific phenotyping beyond the available EHR data for incidental or secondary genetic findings.

CF likely constitutes a gold-standard for the performance of genome-first case identification for monogenetic conditions in population cohorts where the individuals tested have a low prior probability. It is likely that the prospective use of genome-first screening will vary in its diagnostic accuracy and predictive value depending on the cohort and the evidence-base surrounding the chosen gene-condition pair. Importantly, the application of genome-first screening will need to accommodate for additional variables such as age-related penetrance (e.g. for cancer or heart disease) in those gene-condition pairs where it is a major factor and there are no secondary clinical tests of gene function such as the sweat chloride test.

It seems likely that an evidence-based genome-first genecondition pair list will be a more restricted list of gene-condition pairs compared to the list for secondary findings within data generated for unrelated diagnoses. ${ }^{33,34}$ Minimum gene-condition test performance standards may be needed in order to support the employment of genome-first screening outside of the research setting. The development of expert consensus criteria could help establish when a gene-condition pair has the sufficient evidencebase to support population screening.

Since there is an existing population screen for CF that is effective and does not use DNA as the primary screening tool, CF may be amongst the conditions least likely to see significant changes in diagnoses if a DNA-based population screening were pursued. However, using CF as an exemplar in this finding, we demonstrate that an accurate ascertainment of the predictive value of DNA-based screening requires condition-specific phenotyping beyond available EHR data. 


\section{METHODS}

Cohort description

The study cohort consisted of individuals who consented to participate in MyCode, an Institutional Review Board (IRB)-approved program to create a biorepository of blood, serum, and DNA samples for broad research use, including genomic analysis. This study was reviewed by the Geisinger IRB and determined to not be human subjects research as defined in 45CFR46.102(f) in written consent (Study \#2017-054). Data obtained from analysis of MyCode samples is linked to Geisinger EHR; study participants have a median of 14 years of EHR data, providing a longitudinal database of clinical diagnosis, procedures, medications, and laboratory results. As part of the DiscovEHR collaboration between Geisinger and the Regeneron Genetics Center, DNA samples undergo microarray genotyping and whole exome sequencing (WES). The data analyzed for this study consisted of exome variants from the first 50,778 participants, including children. The cohort characteristics have been described previously ${ }^{18}$ and are summarized in Table S1. This study was approved by the Geisinger Institutional Review Board (IRB).

\section{Genotype analysis}

WES for the DiscovEHR collaboration has been described previously. ${ }^{21,35}$ WES variants were confirmed using genotyping data when the markers were available. The same DNA samples were genotyped using the Infinium OmniExpressExome microarray Beadchip (Illumina, San Diego, CA). Following project level quality controls (genotype and sample call rates $>98 \%$, Hardy-Weinberg equilibrium $p>1 \mathrm{e}-06$, and allelic counts greater than or equal to 2), 59,273 samples and 889,966 variants remained for downstream analysis. Of the CFTR variants identified in the patients in the present study, a subset (Q39X, R117H, L206W, G542X, G551D, S1235R, c.489+ $1 \mathrm{G}>\mathrm{T}$, and c.3718-2477 C>T) was available as markers on the BeadChip; all samples with markers on the BeadChip confirmed WES calls, providing a confirmation of the patient genotype status.

\section{Variant annotation and pathogenicity assessment}

Sequence variants were annotated to coding DNA and functional proteins using the NCBI RefSeq Gene definitions, selecting for the transcript with the longest coding sequence among the transcripts with a Locus Reference Genome (LRG) annotation, and excluding transcripts without annotated start and stop codons (SNP \& Variation Suite, Golden Helix, Bozeman, MT). ${ }^{36,37}$ All variants were transcribed to RefSeq NM_000492.3 and translated to NP_000483.3.

The Clinical and Functional Translation of CFTR database (CFTR2, as of 31 August 2018) and the ClinVar database (as of September 15, 2018) were used as the source of validated pathogenic and non-pathogenic CFTR variants. ${ }^{38,39}$

\section{EHR diagnostic code review}

CF ICD10 codes (E84.0, CF with pulmonary manifestations; E84.11, meconium ileus in CF; E84.19, CF with other intestinal manifestations; E84.8, CF with other manifestations; E84.9, CF, unspecified) identified all EHRs among the 50,778 participants who had a relevant CF diagnosis. This list was cross referenced with the list of cases with bi-allelic pathogenic CFTR variants; cases not previously identified through the variant process were sent for open chart review by 2 CF experts (described below) to adjudicate their CF diagnosis.

\section{Clinical open chart review}

Twenty-six cases with either pathogenic bi-allelic CFTR variants identified by DNA analysis (WES plus array), or a CF diagnosis in the EHR without pathogenic bi-allelic CFTR variants underwent open chart review by experienced CF clinicians (CF physician and CF program nurse coordinator). All relevant clinical data were extracted from the EHR, including clinical history as available in progress notes, diagnoses, biochemical parameters, commercial genetic testing, prescription history, spirometry results, and radiographic studies. CF diagnoses was confirmed when an individual had both a clinical presentation of the disease and evidence of CFTR dysfunction or abundant clinical evidence of CF.

CF was also considered present in the following: [1] CFTR dysfunction, defined as sweat chloride $>60 \mathrm{mmol} / \mathrm{L}$, and clinical CFTR genetic analysis showing a combination of two CF-causing CFTR pathogenic variants found in CFTR2 or ClinVar, as per current guidelines from the CF Foundation, [2] if absent sweat chloride testing then CF diagnosis required genetics that were consistent with CF causing pathogenic variants and consistent clinical features, such as longitudinal care by CF physicians, bronchiectasis, and exocrine pancreatic insufficiency. No diagnosis was assumed in those patients lacking sufficient clinical information, irrespective of genetic results.

\section{Concordance of CFTR variant identification in clinic testing and} research exome testing

The list of patients currently managed in the Geisinger CF clinic and their clinical genetic test results were extracted from EHR. The subset who were sequenced and genotyped as volunteers in MyCode were identified. Correlation of variant findings in clinical testing and research sequencing was determined. Clinical genetic testing data was not available on cases of confirmed CF where disease testing and management was carried out at a non-Geisinger facility.

\section{Statistical analysis and graphs}

Standard statistical analyses and all data were plotted using GraphPad Prism (La Jolla, CA).

\section{DATA AVAILABILITY}

All data generated or analyzed during this study are included in this published article (and its supplementary information files).

\section{ACKNOWLEDGEMENTS}

We would like to acknowledge the participants of the MyCode ${ }^{\circledR}$ Community Health Initiative for use of their genomic and electronic health information, without them this study would not be possible. This project is funded, in part, under a Grant with the Pennsylvania Department of Health. The Department specifically disclaims responsibility for any analyses, interpretations or conclusions. The patient enrollment and exome sequencing for the DiscovEHR study were funded by the Regeneron Genetics Center. We thank the Geisinger-Regeneron DiscovEHR Collaboration for making the genotype and phenotype data available for this project.

\section{AUTHOR CONTRIBUTIONS}

J.A.S. and H.M.B. performed open chart review. U.L.M. analyzed the genomic data. J.P. S., U.L.M, M.F.M, and D.J.C. wrote the manuscript. All authors contributed to the study design, development of methodology, interpretation of the data, and reviewing and approving of the final manuscript. D.J.C. and J.A.S. supervised the study. D.J.C., J.A.S., and U.L.M. contributed equally to this work.

\section{ADDITIONAL INFORMATION}

Supplementary information accompanies the paper on the npj Genomic Medicine website (https://doi.org/10.1038/s41525-019-0095-6).

Competing interests: The authors declare no competing interests.

Publisher's note: Springer Nature remains neutral with regard to jurisdictional claims in published maps and institutional affiliations.

\section{REFERENCES}

1. Van Driest, S. L. et al. Association of arrhythmia-related genetic variants with phenotypes documented in electronic medical records. JAMA 315, 47-57 (2016).

2. Haggerty, C. M. et al. Electronic health record phenotype in subjects with genetic variants associated with arrhythmogenic right ventricular cardiomyopathy: a study of 30,716 subjects with exome sequencing. Genet. Med. 19, 1245-1252 (2017).

3. Roden, D. M. Growing pains in cardiovascular genetics. Circulation 138, 1206 (2018).

4. Burke, W. Making sense of the genome remains a work in progress. JAMA 320, 1247-1248 (2018).

5. National Center for Advancing Translational Sciences. Genetic and Rare Diseases Information Center (GARD) https://rarediseases.info.nih.gov/diseases/pages/31/ faqs-about-rare-diseases. 
6. Ong, T. et al. in GeneReviews $((R))$. GeneReviews is a registered trademark of the University of Washington, Seattle. All rights reserved (ed Adam, M. P. et al.) (University of Washington, Seattle, WA, 1993).

7. Alders, M., et al. GeneReviews is a registered trademark of the University of Washington, Seattle. All rights reserved (University of Washington, Seattle, WA, 1993).

8. McNally, E., et al. GeneReviews is a registered trademark of the University of Washington, Seattle. All rights reserved (University of Washington, Seattle, WA, 1993).

9. Farrell, P. M. et al. Diagnosis of cystic fibrosis: consensus guidelines from the cystic fibrosis foundation. J. Pediatr. 181S, S4-S15.e1 (2017).

10. Giovanni, M. A. \& Murray, M. F. Genome-first findings require precision phenotyping. Genet. Med. 20, 1510-1511 (2018).

11. Hamosh, A. et al. Comparison of the clinical manifestations of cystic fibrosis in black and white patients. J. Pediatr. 132, 255-259 (1998)

12. Kosorok, M. R., Wei, W. H. \& Farrell, P. M. The incidence of cystic fibrosis. Stat. Med. 15, 449-462 (1996).

13. Palomaki, G. E., FitzSimmons, S. C. \& Haddow, J. E. Clinical sensitivity of prenatal screening for cystic fibrosis via CFTR carrier testing in a United States panethnic population. Genet. Med. 6, 405-414 (2004).

14. Cystic Fibrosis Foundation. CFF Patient Registry Annual Data Report. (2013).

15. Wagener, J. S., Zemanick, E. T. \& Sontag, M. K. Newborn screening for cystic fibrosis. Curr. Opin. Pediatr. 24, 329-335 (2012).

16. Farrell, P. M., White, T. B., Derichs, N., Castellani, C. \& Rosenstein, B. J. Cystic fibrosis diagnostic challenges over 4 decades: historical perspectives and lessons learned. J. Pediatr. 181S, S16-S26 (2017)

17. Schwartz, M. L. B. et al. A model for genome-first care: returning secondary genomic findings to participants and their healthcare providers in a large research cohort. Am. J. Hum. Genet. 103, 328-337 (2018).

18. Carey, D. J. et al. The Geisinger MyCode community health initiative: an electronic health record-linked biobank for precision medicine research. Genet. Med. 18, 906-913 (2016).

19. Abul-Husn, N. S. et al. A protein-truncating HSD17B13 variant and protection from chronic liver disease. N. Engl. J. Med. 378, 1096-1106 (2018).

20. Manickam, K. et al. Exome sequencing-based screening for BRCA1/2 expected pathogenic variants among adult biobank participants. JAMA Netw. Open 1, e182140 (2018)

21. Dewey, F. E. et al. Distribution and clinical impact of functional variants in 50,726 whole-exome sequences from the DiscovEHR study. Science 354 https://doi.org/ 10.1126/science.aaf6814 (2016)

22. Lee, M. et al. Systematic computational identification of variants that activate exonic and intronic cryptic splice sites. Am. J. Hum. Genet. 100, 751-765 (2017).

23. Simon, D. \& Boring, J. R., III. in Clinical Methods: The History, Physical, and Laboratory Examinations (eds 3rd, Walker, H. K., Hall, W. D. \& Hurst, J. W.) (Butterworth Publishers, a division of Reed Publishing, Boston, 1990).

24. Kloosterboer, M. et al. Clarification of laboratory and clinical variables that influence cystic fibrosis newborn screening with initial analysis of immunoreactive trypsinogen. Pediatrics 123, e338-e346 (2009).

25. Castellani, C. et al. Consensus on the use and interpretation of cystic fibrosis mutation analysis in clinical practice. J. Cyst. Fibros. 7, 179-196 (2008).

26. Vernooij-van Langen, A. M. et al. Novel strategies in newborn screening for cystic fibrosis: a prospective controlled study. Thorax 67, 289-295 (2012).
27. Meagher, K. M. \& Berg, J. S. Too much of a good thing? Overdiagnosis, or overestimating risk in preventive genomic screening. Pers. Med. 15, 343-346 (2018).

28. Rehm, H. L. et al. ClinGen-the clinical genome resource. N. Engl. J. Med. 372, 2235-2242 (2015).

29. Hennekam, R. C. \& Biesecker, L. G. Next-generation sequencing demands nextgeneration phenotyping. Hum. Mutat. 33, 884-886 (2012).

30. Gibson, L. E. \& Cooke, R. E. A test for concentration of electrolytes in sweat in cystic fibrosis of the pancreas utilizing pilocarpine by iontophoresis. Pediatrics 23, 545-549 (1959).

31. Collie, J. T., Massie, R. J., Jones, O. A., LeGrys, V. A. \& Greaves, R. F. Sixty-five years since the New York heat wave: advances in sweat testing for cystic fibrosis. Pediatr. Pulmonol. 49, 106-117 (2014).

32. Tsui, L. C. \& Dorfman, R. The cystic fibrosis gene: a molecular genetic perspective. Cold Spring Harb. Perspect. Med. 3, a009472 (2013).

33. Kalia, S. S. et al. Recommendations for reporting of secondary findings in clinical exome and genome sequencing, 2016 update (ACMG SFv2.0): a policy statement of the American College of Medical Genetics and Genomics. Genet. Med. 19, 249-255 (2017).

34. Murray M. F. et al. A Proposed Approach for Implementing Genomics-Based Screening Programs for Healthy Adults. 1-16 (National Academy of Medicine, Washington, DC, 2018).

35. Mirshahi, U. L. et al. Trajectory of exonic variant discovery in a large clinical population: implications for variant curation. Genet. Med. 21, 1417-1424 (2019).

36. Richards, S. et al. Standards and guidelines for the interpretation of sequence variants: a joint consensus recommendation of the American College of Medical Genetics and Genomics and the Association for Molecular Pathology. Genet. Med. 17, 405-424 (2015)

37. Dalgleish, R. et al. Locus Reference Genomic sequences: an improved basis for describing human DNA variants. Genome Med. 2, 24 (2010).

38. The Clinical and Functional TRanslation of CFTR (CFTR2); available at http://cftr2. org.

39. Sosnay, P. R. et al. Applying cystic fibrosis transmembrane conductance regulator genetics and CFTR2 data to facilitate diagnoses. J. Pediatr. 181S, S27-S32.e1 (2017).

Open Access This article is licensed under a Creative Commons Attribution 4.0 International License, which permits use, sharing, adaptation, distribution and reproduction in any medium or format, as long as you give appropriate credit to the original author(s) and the source, provide a link to the Creative Commons license, and indicate if changes were made. The images or other third party material in this article are included in the article's Creative Commons license, unless indicated otherwise in a credit line to the material. If material is not included in the article's Creative Commons license and your intended use is not permitted by statutory regulation or exceeds the permitted use, you will need to obtain permission directly from the copyright holder. To view a copy of this license, visit http://creativecommons. org/licenses/by/4.0/.

(c) The Author(s) 2019 